\title{
Fatores de risco cardiovasculares em crianças obesas
}

\author{
Cardiovascular risk factors in obese children
}

\author{
Fernanda M. Gazolla*, Maria Alice N. Bordallo, Isabel R. Madeira, Cecília N. M. Carvalho, \\ Paulo F. Collett-Solberg, Ana Paula N. Bordallo, Clarice B. de Medeiros, Carolina B. da Cunha
}

\begin{abstract}
Resumo
Desde os anos pré-púberes, crianças com excesso de peso são expostas precocemente a fatores de risco cardiovascular que atuam de forma negativa no endotélio vascular e na íntima-média dos vasos, levando ao início da doença aterosclerótica nessa faixa etária. Esta revisão tem por objetivo trazer ao conhecimento dos profissionais de saúde a importância da identificação e da intervenção terapêutica precoces em crianças com excesso de peso, sabidamente em risco para a instalação da síndrome metabólica, na tentativa de evitar a evolução para doenças crônicas, em especial para as doenças cardiovasculares. Atualmente a obesidade atinge todas as faixas etárias da população e, assim como nos adultos, vem alcançando proporções epidêmicas nas crianças e adolescentes, já sendo considerada um dos desafios mais sérios de saúde pública no século XXI. Ao contrário do que se acreditava, que crianças obesas não apresentavam risco cardiovascular até atingirem a idade adulta, os estudos apontam que a aterosclerose se inicia na infância. A constatação de que o excesso de peso na infância e os fatores de risco a ele relacionados persistem até a vida adulta foi documentada em ao menos três grandes estudos de coorte: o Muscatine Study, o Cardiovascular Risk in Young Finns Study e o Bogalusa Heart Study. O tempo de exposição aos múltiplos fatores de risco nessa etapa da vida até a adultícia parece estar diretamente associado à morbimortalidade por doença cardiovascular. A demonstração de que as doenças cardiovasculares podem ter sua origem na infância leva à necessidade de que seus fatores de risco sejam amplamente investigados nesse período, com o objetivo de planejar intervenções cada vez mais precoces e possivelmente mais efetivas, reduzindo no futuro a morbimortalidade por doenças cardiovasculares.
\end{abstract}

Descritores: Criança; Obesidade; Fatores de risco; Doenças cardiovasculares.

\begin{abstract}
Prepubertal children with excess weight are already exposed to cardiovascular risk factors that affect negatively the vascular endothelium and the intima media of the blood vessels inducing atherosclerotic changes. The objective of this review is to bring to the attention of health professionals the importance of early identification and intervention in children with excess weight, a known risk factor for metabolic syndrome, with the goal of preventing the development of chronic diseases, especially cardiovascular diseases. Obesity affects people of all ages and is considered an epidemic in children and adolescents, already the most important health challenge of the 21st century. Previously considered an adult disease, atherosclerosis is now believed to initiate during childhood. The proof that excess weight and its cardiovascular risk factors persist to adulthood came from at least three large cohort studies: the Muscatine Study, the Cardiovascular Risk in Young Finns Study, and the Bogalusa Heart Study. The length of time exposed to the multiple risk factors during this stage of life seems to be directly associated to cardiovascular disease morbimortality. The demonstration that cardiovascular diseases may have their origin during childhood creates the necessity to investigate their risk factors during this period of time with the goal to plan earlier and, possibly, more effective interventions to reduce in the future the cardiovascular disease morbimortality.
\end{abstract}


Keywords: Child; Obesity; Risk factors; Cardiovascular diseases.

\section{Introdução}

As doenças cardiovasculares (DCV) são atualmente a principal causa de morte no mundo, tanto nos países desenvolvidos como naqueles em desenvolvimento. 'Estudos apresentados nas últimas três décadas têm mostrado que a probabilidade de algum evento cardiovascular ocorrer aumenta na presença de múltiplos fatores de risco estabelecidos para aterosclerose. ${ }^{2}$

Em geral, as manifestações clínicas das DCV acontecem na vida adulta, no entanto, estudos atuais, como o de McGill e colaboradores, ${ }^{3}$ indicam que o processo aterosclerótico começa a se desenvolver na primeira década de vida. Há evidências de que este processo progride com a idade e exibe gravidade diretamente proporcional ao número de fatores de risco cardiovascular (FRCV) agregados. Os FRCV tendem a se agrupar em indivíduos e interagem sinergicamente aumentando o risco cardiovascular com o passar dos anos. ${ }^{4}$

A partir do conhecimento de que este efeito sinérgico é determinante para o desenvolvimento da doença aterosclerótica desde os anos pré -púberes até a adultícia, houve um crescente interesse da comunidade científica nas últimas três décadas em entender melhor a agregação e sinergismo dos FRCV tradicionais e a utilidade dos marcadores de risco não tradicionais na prática clínica. A intenção é que este conhecimento possa ajudar no desenvolvimento de estratégias terapêuticas para prevenir DCV precocemente.

Com base nas evidências do desenvolvimento silencioso da doença aterosclerótica e do aumento alarmante do excesso de peso na infância e dos FRCV a ele associados, esta revisão tem por objetivo trazer ao conhecimento dos profissionais de saúde a importância da identificação e intervenção terapêutica daquelas crianças em risco de instalação da síndrome metabólica, o mais precocemente possível, a fim de retardar ou bloquear a evolução para as DCV e doenças crônicas não comunicáveis a ela relacionadas.

\section{Considerações gerais}

O conceito de FRCV foi introduzido nos estu- dos iniciais do Framingham Heart Study, em 1961. Estes estudos ligavam a presença de condições clínicas prévias como dislipidemia, hipertensão arterial sistêmica (HAS) e diabetes mellitus (DM), com DCV futura. ${ }^{5}$

O risco é avaliado com base no somatório das características (fatores de risco) que aumentam a probabilidade de um indivíduo exposto e livre de doença vir a desenvolvê-la. ${ }^{6}$

\section{Fatores de risco cardiovascular (FRCV)}

Os FRCV são divididos em duas grandes categorias: os tradicionais, sendo estes modificáveis ou não, e os não tradicionais. ${ }^{7}$ Dentro da categoria de tradicionais não modificáveis, são reconhecidos na literatura idade, sexo e histórico familiar precoce de DCV, enquanto dislipidemia, HAS, DM, tabagismo, sedentarismo e obesidade representam a categoria de tradicionais modificáveis. ${ }^{8} \mathrm{Um}$ grupo emergente de marcadores (denominado marcadores de risco cardiovascular) é identificado como não tradicional. Dentre os mais estudados estão as citocinas inflamatórias, como a proteína C-reativa (PCR) e a interleucina-6 (IL-6), as adipocinas, como a leptina e a adiponectina, e a avaliação do complexo médio-intimal das artérias de grande calibre. ${ }^{9}$

\section{Fatores de risco cardiovascular tradicionais não modificáveis}

\section{Idade, sexo e histórico familiar de DCV precoce}

Em relação aos FRCV tradicionais não modificáveis há dois pontos bem determinados: os riscos para as DCV aumentam com a idade, sendo que a cada dez anos há uma possibilidade de aumentar em 2,5 vezes a mortalidade por essas doenças, e a magnitude dos fatores de risco e a ocorrência de manifestações clínicas aparecem mais tardiamente em mulheres do que em homens. ${ }^{10}$ Com relação à herança genética, estudos mostram que crianças e adolescentes com histórico familiar de DCV prematura, ou seja, a presença de um evento cardiovascular em ascendentes antes dos 55 e 65 anos de idade, respectivamente para o sexo 
masculino e feminino, apresentam um ou mais fatores de risco para a aterosclerose."

\section{Fatores de risco cardiovascular tradicionais modificáveis}

\section{Obesidade}

De forma semelhante ao que anteriormente já havia acontecido com os países desenvolvidos, um fenômeno conhecido como transição nutricional vem ocorrendo, nas últimas três décadas, no Brasil e em outros países em desenvolvimento. Estudos populacionais têm demonstrado claramente o declínio dos casos de desnutrição e o aumento dos casos de obesidade em diversos países. $\mathrm{O}$ conceito de transição nutricional refere-se às mudanças, através dos séculos, do padrão alimentar das populações. Estas mudanças são decorrentes da ocidentalização dos cardápios e das escolhas alimentares de vários países, independentemente de suas riquezas. Pontos comuns deste efeito são uma dieta rica em gorduras, açúcares simples e alimentos refinados, e pobre em fibras e açúcares complexos, como o amido. ${ }^{12}$

Neste cenário observa-se paralelamente ao aumento da urbanização, o aumento da disponibilidade de alimentos, do sedentarismo e, consequentemente, da prevalência da obesidade. ${ }^{13}$ Este fato é bastante preocupante, pois a associação da obesidade com outros FRCV, até alguns anos atrás, era mais evidente em adultos. No entanto, hoje já pode ser observada frequentemente na faixa etária mais jovem. ${ }^{14}$

A obesidade é um distúrbio nutricional e metabólico que cursa com aumento da massa adiposa do organismo, cuja fisiopatologia ainda não é claramente conhecida. É resultante de um desequilíbrio entre ingestão e consumo energético, e a etiologia é multifatorial, sendo provável a herança poligênica, onde os fatores genéticos têm papel importante e os ambientais funcionariam como agravantes. ${ }^{15}$

A complicação mais grave relacionada à obesidade são as DCV. A obesidade afeta a saúde cardiovascular e favorece o desenvolvimento da aterosclerose por diversas vias. $\mathrm{O}$ aumento do tecido adiposo parece ser o primeiro passo na promoção das alterações no metabolismo da glicose e no desenvolvimento de resistência à insulina. ${ }^{16}$ A obesidade gera resistência insulínica, enquanto esta parece exacerbar os efeitos adversos da obesidade. Até o momento não está claro se um estado de resistência insulínica predeterminado geneticamente surge primeiro, ou se o evento inicial é a obesidade. ${ }^{17} \mathrm{Na}$ faixa etária pediátrica, a obesidade parece ser importante gatilho de resistência insulínica,,$^{17}$ o que torna as crianças obesas um grupo de risco, levando à busca de indicadores clínicos e laboratoriais neste grupo por parte da comunidade científica.

As complicações metabólicas da obesidade em adultos e crianças não se relacionam somente ao excesso de peso, mas também à distribuição da gordura corporal. Mais do que a quantidade de gordura, sua distribuição desempenha papel essencial sobre a ação da insulina. ${ }^{18}$

\section{Síndrome metabólica e resistência insulínica}

O conceito de síndrome metabólica (SM), introduzido por Gerald Reaven, em 1988, descreve o conjunto de alterações metabólicas e hemodinâmicas geradas pela presença de resistência insulínica que, quando presentes no mesmo indivíduo, aumentam o risco de DCV. Cada alteração isoladamente é um fator de risco tradicional modificável para DCV, porém quando combinados, seus efeitos se potencializam. Nessa síndrome, a resistência insulínica e a hiperinsulinemia explicam a relação entre a obesidade e as outras anormalidades. ${ }^{19}$

A prevalência da SM está aumentando em todo o mundo, acompanhando a epidemia global de obesidade. Se antes era considerada uma síndrome tipicamente do adulto, com o aumento das taxas de obesidade infantil e em adolescentes, a presença de componentes da SM ocorre em idades cada vez mais tenras. ${ }^{20-22}$

A patogênese da agregação dos componentes da SM ainda não está totalmente conhecida e, até o momento, não se encontrou um mecanismo único. Acredita-se que a resistência insulínica e o excesso de tecido adiposo visceral (denominado obesidade visceral) sejam os mecanismos iniciais mais importantes. A resistência insulínica é descrita como central na patogênese da síndrome, explicando a associação entre obesidade e dis- 
função vascular. ${ }^{23}$

Da mesma forma que para adultos, não há uma definição oficial de SM para crianças, e as mesmas limitações e dificuldades em relação à uniformidade dos critérios diagnósticos existem. No entanto, seus principais componentes e marcadores clínicos já foram razoavelmente estabelecidos na faixa etária pediátrica. A International Diabetes Federation (IDF), em 2007, publicou uma definição de SM para crianças e adolescentes. Os critérios, no entanto, só devem ser aplicados na clínica a crianças acima de dez anos, por falta de evidências científicas referentes aos mais jovens. ${ }^{24}$

Independentemente da definição utilizada para o diagnóstico da SM, a maioria concorda quanto aos componentes centrais que devem estar presentes - obesidade, dislipidemia, HAS e intolerância à glicose -, mas discordam acerca dos pontos de corte de cada componente individual, de requisitos específicos (por exemplo, obesidade visceral ou resistência insulínica) e na inclusão de fatores adicionais (por exemplo, microalbuminúria). ${ }^{25}$

\section{Fatores de risco cardiovascular não tradicionais}

\section{Citocinas inflamatórias, adipocinas e} avaliação do complexo médio-intimal de artérias

Os marcadores de risco cardiovascular não tradicionais, recém-identificados, estão sendo intensamente investigados nos últimos anos e ainda não têm seu papel clínico bem definido, por isso o seu uso não é indicado na rotina clínica.

É necessário distinguir o conceito de fator de risco com o de marcador de risco. No primeiro caso, já está estabelecida a relação fisiopatológica entre o fator de risco e a doença, e no segundo caso, há apenas uma associação que indica maior risco, porém sem causalidade estabelecida. O estudo destes novos marcadores de risco pode vir a contribuir para o melhor entendimento da gênese das DCV, e eles podem constituir elementos valiosos de estratificação de uma população de crianças em maior risco para DCV, uma vez que muitos dos FRCV tradicionais ainda não estão presentes na infância, como HAS e DM. ${ }^{26}$

Com o crescente número de estudos a respeito da importância da obesidade infantil na gênese das DCV, várias substâncias produzidas no tecido adiposo, as adipocinas, e outras com papel na inflamação e no estresse oxidativo, são candidatas a marcadores de risco. O conhecimento a este respeito poderá, eventualmente, conduzir a estratégias terapêuticas direcionadas a prevenção de DCV em indivíduos cada vez mais jovens. ${ }^{27}$

O excesso de gordura corporal não só conduz às alterações metabólicas do tecido adiposo e à indução de resistência insulínica, mas também à disfunção endotelial, por meio de efeitos pró -inflamatórios e pró-trombóticos decorrentes da ação das citocinas inflamatórias e adipocinas. Foi a partir dessas descobertas que o tecido adiposo passou a não ser mais considerado um tecido apenas de depósito, surgindo uma nova maneira de abordar a obesidade: como uma doença inflamatória. Neste caso, podemos considerar a célula adiposa como "disfuncional", secretante de diversas substâncias bioativas como as adipocinas e citocinas inflamatórias, que vão afetar a sensibilidade à insulina e a função endotelial. ${ }^{28}$

Diversas adipocinas secretadas pelos adipócitos aumentam, direta ou indiretamente, a produção e a circulação de marcadores relacionados com a inflamação. Existem evidências demonstrando que o estado inflamatório pode ser devido à resistência insulínica e às outras desordens associadas à obesidade, como os outros componentes da SM. Assim, aventa-se a hipótese de que a inflamação seja uma consequência da obesidade. $^{29}$

Algumas adipocinas importantes na inter-relação do sistema vascular do estado inflamatório crônico e da obesidade são: a leptina (tem relação direta com a massa corporal e papel importante na regulação desta e do apetite), a adiponectina (tem relação inversa com a massa corporal e propriedades anti-inflamatórias), a IL-6 (aumenta a produção da PCR pelo fígado) e a PCR (molécula pró-inflamatória). ${ }^{9}$

A injúria endotelial constitui o evento inicial do processo de formação da placa aterosclerótica, e a aterogênese pode ser considerada como uma resposta inflamatória protetora à agressão ao endotélio. São alterações precoces e "silenciosas" do processo aterogênico, marcadoras 
de dano arterial, e que precedem a formação da placa ateromatosa. Essas alterações ocorrem muitos anos antes das manifestações estruturais e clínicas da doença, verificando-se, portanto, a existência de um longo período assintomático. $\mathrm{O}$ conceito de que a aterosclerose é um fenômeno crônico degenerativo do idoso foi substituído pelo que a considera uma doença inflamatória crônica subclínica iniciada na infância. ${ }^{30}$

A disfunção endotelial, associada à vasodilatação endotélio-dependente prejudicada, à redução da complacência arterial e ao espessamento da camada íntima-média carotídea, é evento precoce na evolução da aterosclerose em indivíduos com obesidade e que já pode ser vista em crianças de 9 a 12 anos de idade. A disfunção do endotélio, ao longo do tempo, leva ao espessamento mensurável da íntima-média da parede de artérias de grande e médio calibre, como aorta, carótidas e ilíacas. Este espessamento é considerado a primeira alteração anatômica da aterosclerose. ${ }^{31}$

A associação do espessamento da íntimamédia carotídea com fatores de risco conhecidos para DCV, como hipercolesterolemia familiar, HAS e DM2, já vem sendo demonstrada em crianças, adolescentes e adultos jovens. ${ }^{32}$

Alguns grupos de pesquisadores têm lançado esforços na tentativa de encontrar "a idade de risco", em que as alterações vasculares estruturais poderiam ser identificadas de forma confiável, e principalmente, a idade em que as artérias dessas crianças já estivessem sobre a influência dos FRCV. No ano de 2010, Juonala e colaboradores ${ }^{33}$ apontaram, a partir de análise de dados de quatro grandes estudos populacionais e de coorte (o Muscatine Study, o Cardiovascular Risk in Young Finns Study, o Bogalusa Heart Study e o Childhood Determinants of Adult Health Study), que a presença dos FRCV dos nove anos de idade em diante seria preditiva de doença aterosclerótica subclínica na idade adulta, sugerindo que estes fatores fossem mensurados a partir dos nove anos de idade.

\section{Conclusão}

Com base no que já se conhece do processo de desenvolvimento da aterosclerose, sem dúvida, o controle dos FRCV em crianças pré-púberes é mandatório para a prevenção das DCV e das outras doenças associadas a esses fatores.

O rápido aumento da prevalência da obesidade em crianças cada vez mais jovens, nas últimas décadas, pode eventualmente estar antecipando a idade de início da instalação da aterosclerose. Aliado a isto, a agregação dos FRCV e a exposição a estes, bem como a sua perpetuação da infância à adultícia, estariam levando ao aumento da incidência de DCV em todo o mundo.

A intervenção sobre os fatores de risco detectados determina mudanças benéficas no perfil de risco identificado, mesmo nessa fase, principalmente por meio do envolvimento das crianças e adolescentes nas abordagens educativas em saúde. Estratégias desta natureza são importantes uma vez que os fatores de risco identificados em crianças e adolescentes obesos tendem a persistir na vida adulta, concorrendo para o estabelecimento das DCV cada vez mais precocemente.

\section{Referências}

1. World Health Organization. The Top 10 Causes of Death. Fact sheet $N^{\circ} 310$. Geneva: World Health Organization; 2013.

2. Tolfrey K. Intraindividual variability of children's blood, lipid and lipoprotein concentrations: a review. Prev Cardiol. 2002 Summer;5(3):145-51. http://dx.doi. org/10.1111/j.1520-037X.2002.00563.x

3. McGill HC, McMahan CA, Zieske AW, Sloop GD, Walcott JV, Troxclair DA, et al. Associations of coronary heart disease risk factors with the intermediate lesion of atherosclerosis in youth. The Pathobiological Determinants of Atherosclerosis in Youth (PDAY) Research Group. Arterioscler Thromb Vasc Biol. 2000 Aug;20(8):1998-2004. http://dx.doi. org/10.1161/01.ATV.20.8.1998

4. Berenson GS, Srinivasan SR, Bao W, Newman WP 3rd, Tracy RE, Wattigney WA. Association between multiple cardiovascular risk factors and atherosclerosis in children and young adults. Bogalusa Heart Study. N Engl J Med. 1998 Jun 4;338(23):1650-6. http://dx.doi.org/10.1056/NEJM199806043382302

5. Kannel WB, Dawber TR, Kagan A, Revotskie N, Stokes J 3rd. Factors of risk in the development of coronary heart disease-six year follow-up experience: the Framingham Study. Ann Intern Med. 1961 Jul;55(1):33-50. http://dx.doi.org/10.7326/0003-481955-1-33

6. Almeida Filho N, Rouquayrol MZ. Introdução à epidemiologia. 3a ed. Rio de Janeiro: MEDSl; 2002. 293 p.

7. McGill HC Jr, McMahan CA, Gidding SS. Preventing heart disease in the 21st century: implications of 
the Pathobiological Determinants of Atherosclerosis in Youth (PDAY) Study. Circulation. 2008 Mar;117(9):1216-27. http://dx.doi.org/10.1161/CIRCULATIONAHA.107.717033

8. D’Agostino RB Sr, Vassan RS, Pencina MJ, Wolf PA, Cobain M, Massaro JM, et al. General cardiovascular risk profile for use in primary care: the Framingham Heart Study. Circulation. 2008 Feb 12;117(6):74353. http://dx.doi.org/10.1161/CIRCULATIONAHA.107.699579

9. U.S. Preventive Services Task Force. Using nontraditional risk factors in coronary heart disease risk assessment: U.S. Preventive Services Task Force recommendation statement. Ann Intern Med. 2009 Oct 6;151(7):474-82. http://dx.doi.org/10.7326/00034819-151-7-200910060-00008

10. Rabelo LM. Fatores de risco para doença aterosclerótica na adolescência. J Pediatr. 2001 Nov/ Dez;77(Supl.2):S153-64. http://dx.doi.org/10.2223/ JPED.303

11. Romaldini CC, Issler H, Cardoso AL, Diament J, Forti N. Fatores de risco para aterosclerose em crianças e adolescentes com história familiar de doença arterial coronariana prematura. J Pediatr. 2004 Março/Abril;80(2):135-40. http://dx.doi.org/10.2223/ JPED.1153

12. Popkin BM, Keyou G, Zhai F, Guo X, Ma H, Zohoori N. The nutrition transition in China: a cross-sectional analysis. Eur J Clin Nutr. 1993 May;47(5):333-46.

13. Monteiro CA, Mondini L, Souza ALM, Popkin BM. Da desnutrição para a obesidade: a transição nutricional no Brasil. In: Monteiro CA (org.). Velhos e novos males da saúde no Brasil: a evolução do país e de suas doenças. São Paulo: Hucitec; 1995. p. 247-55.

14. Styne DM. Childhood and adolescent obesity: prevalence and significance. Pediatr Clin North Amer. 2001 Aug;48(4):823-54.

15. Jebb SA. Aetiology of obesity. Br Med Bull. 1997;53(2):264-85.

16. McGarry JD, Dobbins RL. Fatty acids, lipotoxicity and insulin secretion. Diabetologia. 1999 Feb;42(2):12838. http://dx.doi.org/10.1007/s001250051130

17. Ten S, MacLaren N. Insulin resistance syndrome in children. J Clin Endocrinol Metab. 2004 Jun;89(6):2526-39. http://dx.doi.org/10.1210/jc.20040276

18. Daniels SR, Morrison JA, Sprecher DL, Khoury P, Kimball TR. Association of body fat distribution and cardiovascular risk factors in children and adolescents. Circulation. 1999 Feb 2;99(4):541-5. http:// dx.doi.org/10.1161/ 01.CIR.99.4.54

19. Reaven GM. Role of insulin resistance in human disease. Diabetes. 1988 Dec;37(12):1595-607. http:// dx.doi.org/10.2337/diab.37.12.1595
20.McCrindle BW. Cardiovascular consequences of paediatric obesity: will there be a future epidemic of premature cardiovascular disease? Paediatr Child Health. 2007 Mar;12(3):175-7.

21. Madeira IR, Bordallo MA, Carvalho CN, Gazolla FM, de Souza FM, de Matos HJ, et al. The role of metabolic syndrome components and adipokines in insulin resistance in prepubertal children. J Pediatr Endocr Metab. 2011;24(5-6):289-95.

22. Madeira IR, Carvalho CN, Gazolla FM, Pinto LW, Borges MA, Bordallo MA. Impact of obesity on metabolic syndrome components and adipokines in prepubertal children. J Pediatr (Rio J). 2009 May/ June;85(3):261-8. http://dx.doi.org/10.2223/JPED.1873

23. Aggoun Y. Obesity, metabolic syndrome, and cardiovascular disease. Pediatr Research. 2007 Jun;61(6):653-9. http://dx.doi.org/10.1203/pdr.0b013e31805d8a8c

24. Zimmet P, Alberti KG, Kaufman F, Tajima N, Silink $\mathrm{M}$, Arslanian S, et al. The metabolic syndrome in children and adolescents - an IDF consensus report. Pediatr Diabetes. 2007 Oct;8(5):299-306. http://dx. doi.org/10.1111/j.1399-5448.2007.00271.x

25. Levesque J, Lamarche B. The metabolic syndrome: definitions, prevalence and management. J Nutrigenet Nutrigenomics. 2008 Feb;1(3):100-8. http://dx.doi. org/10.1159/000112457

26. May A, Wang TJ. Biomarkers for cardiovascular disease: challenges and future directions. Trends Mol Med. 2008 Jun;14(6):261-7. http://dx.doi.org/10.1016/j. molmed.2008.04.003

27. Hlatky MA, Greenland P, Arnett DK, Ballantyne CM, Criqui MH, Elkind MS, et al. Criteria for evaluation of novel markers of cardiovascular risk: a scientific statement from the American Heart Association. Circulation. 2009 May 5;119(17):2408-16. http://dx.doi. org/10.1161/CIRCULATIONAHA.109.192278

28. Beauloye V, Zech F, Tran HT, Clapuyt P, Maes M, Brichard SM. Determinants of early atherosclerosis in obese children and adolescents. J Clin Endocrinol Metab. 2007 Aug;92(8):3025-32. http://dx.doi. org/10.1210/jc.2007-0619

29. Trayhurn P. Adipocyte biology. Obes Rev. 2007 Mar;8(suppl 1):41-4. http://dx.doi.org/10.1111/j.1467789X.2007.00316.x

30. Celermajer DS, Sorensen KE, Gooch VM, Spiegelhalter DJ, Miller OI, Sullivan ID, et al. Non-invasive detection of endothelial dysfunction in children and adults at risk of atherosclerosis. Lancet. $1992 \mathrm{Nov}$ 7;340(8828):1111-5.

31. Slyper AH. Clinical review 168: what vascular ultrasound testing has revealed about pediatric atherogenesis, and a potential clinical role for ultrasound in pediatric risk assessment. J Clin Endocrinol Metab. 
2004 Jul;89(7):3089-95. http://dx.doi.org/10.1210/ jc.2003-030644

32. Urbina EM, Williams RV, Alpert BS, Collins RT, Daniels SR, Hayman L, et al. Noninvasive assessment of subclinical atherosclerosis in children and adolescents: recommendations for standard assessment for clinical research - a scientific statement from the American Heart Association. Hypertension. 2009 Nov;54(5):919-50. http://dx.doi.org/10.1161/HYPERTENSIONAHA.109.192639
33. Juonala M, Magnussen CG, Venn A, Dwyer T, Burns TL, Davis PH, et al. Influence of age on associations between childhood risk factors and carotid intima-media thickness in adulthood: the cardiovascular risk in Young Finns Study, The Childhood Determinants of Adult Health Study, The Bogalusa Heart Study, and The Muscatine Study for The International Childhood Cardiovascular Cohort (i3c) Consortium. Circulation. 2010 Dec;122(24):2514-20. http:// dx.doi.org/10.1161/CIRCULATIONAHA.110.966465

Recebido: $\quad$ 06/08/2013.

Revisado: $\quad$ 26/11/2013.

Aprovado: $\quad$ 27/01/2014. 